\title{
Le statut du rapport dans l'audit organisationnel : de sa légitimation par l'écriture. à son action comme agent non humain
}

The Status of the Report in Organizational Auditing: From Legitimization

through Writing to Action as a Nonhuman Actor

François Lambotte et Philippe Scieur

\section{OpenEdition}

Journals

Édition électronique

URL : http://journals.openedition.org/edc/5154

DOI : 10.4000/edc.5154

ISSN : 2101-0366

Éditeur

Université Lille-3

Édition imprimée

Date de publication : 1 juin 2013

Pagination : 111-128

ISBN : 978-2-917562-09-3

ISSN : $1270-6841$

\section{Référence électronique}

François Lambotte et Philippe Scieur, « Le statut du rapport dans l'audit organisationnel : de sa légitimation par l'écriture. à son action comme agent non humain », Études de communication [En ligne], 40 | 2013, mis en ligne le 01 juin 2013, consulté le 19 avril 2019. URL : http:// journals.openedition.org/edc/5154; DOI : 10.4000/edc.5154

Ce document a été généré automatiquement le 19 avril 2019

(c) Tous droits réservés 


\title{
Le statut du rapport dans l'audit organisationnel : de sa légitimation par l'écriture. à son action comme agent non humain
}

\author{
The Status of the Report in Organizational Auditing: From Legitimization \\ through Writing to Action as a Nonhuman Actor
}

François Lambotte et Philippe Scieur

1 Cet article porte sur le statut du rapport dans l'audit organisationnel. Elle met l'emphase sur (1) la construction de la légitimation de l'audit à travers son écriture, fruit d'une construction interactive entre les acteurs concernés par la convention, et sur (2) son rôle d'interactant non humain, au sens de la sociologie de la traduction, qui prolonge l'interaction entre les acteurs temporellement au-delà de la remise finale du rapport au commanditaire. Le cadre théorique de notre propos s'inscrit dans le paradigme épistémologique constructiviste non radical, tant dans sa perspective individualiste que dans celle qui relève de l'interactionnisme.

2 Beaucoup d'auteurs d'ouvrages à destination de praticiens se sont penchés sur l'audit organisationnel notamment basé sur la théorie de la systémique (par exemple De SaintGeorges, 2000 ; Lambotte et Lafrance, 2011 ; Mucchielli, 2004, 2005). Ces auteurs mettent en avant les avantages et les inconvénients des approches socio-constructivistes dans l'élaboration d'un diagnostic permettant d'identifier et de distinguer les sources et les conséquences d'un dysfonctionnement organisationnel au sein d'un département, voire dans l'ensemble de l'organisation. Parmi les chercheurs en organisation, peu d'auteurs ${ }^{1}$ se sont intéressés au statut même de l'intervention et de l'intervenant (système intervenant) et de son incidence sur l'organisation (système client analysé) (De SaintGeorges, 2000). Or, on sait qu'il y a des risques liés à l'audit : ses effets peuvent se limiter à perturber l'organisation au moment où les intervenants procèdent à leur investigation, par entretien ou observation, et le diagnostic formulé peut être rapidement oublié et les 
solutions préconisées jamais mises en œuvre. De Saint Georges (2000) nous sensibilise à cette question en mettant l'accent sur le choix préalable du type d'intervention entre recherche, audit, formation et intervention clinique. Nous mettrons ici la focale sur la transition de l'audit vers la formation ou vers l'intervention clinique. Plus précisément, nous souhaitons questionner le rôle que peut jouer l'écriture et la négociation du rapport d'audit dans ce passage d'un audit à une autre forme d'intervention qui prolonge le travail réalisé.

Nous pensons que le rapport est un objet central de l'audit car il rend visible les problèmes vécus par les membres du personnel à une hiérarchie trop éloignée du terrain (Pène, 1995). Comme Anderson (2004), dont l'analyse porte sur l'écriture d'un projet de changement ou Robichaud et Benoit-Barné (2010) qui analysent l'écriture d'un document préparant la planification stratégique, nous avançons que le contexte et les acteurs participant au dispositif d'écriture du rapport (Delcambre, 1990b) auront une incidence non seulement sur le contenu même du rapport mais également sur sa légitimité et son appropriation, comme agent non humain, par les acteurs concernés de l'organisation auditée. De plus, nous montrons dans cet article que le rapport, parce qu'il fait partie de l'organisation, agit dans le processus d'amélioration de l'organisation et prolonge la relation entre le système intervenant et le système client, paradoxalement en dehors d'une logique instrumentale a prioristique.

Pour réaliser cette démonstration, nous analysons la construction d'un rapport d'audit à travers l'analyse des écrits dont nous sommes co-auteurs en tant qu'intervenants dans l'organisation auditée. Fruit d'une fertilisation croisée entre théories et pratique, cet article tend à montrer que le socio-constructivisme se situe au-delà d'une idéologie d'intervention.

\section{La textualisation : objet d'analyse}

5 Notre réflexion porte donc sur la textualisation. Celle-ci est le processus qui relie conversations et textes. C'est le cycle à travers lequel des conversations se traduisent en un texte et inversément comment un texte se traduit dans des actes de conversations (Taylor et al., 1996). Ce processus met l'accent dans un premier temps sur la négociation du sens, la production, à travers les conversations, d'un niveau plus général ou collectif de signification (Fayad et Lambotte, 2011) qui peut prendre la forme d'une histoire ou d'un écrit (Anderson, 2004 ; Robichaud et al., 2004). Comme l'exprime Sophie Pène :

Les formes écrites sont insérées dans de multiples rebonds...ces situations de communication successives et interdépendantes, ne déterminent pas simplement des effets de copiage, de citation, de répétition. Le traitement sémiotique proprement dit est affecté par la variété des formes et des moments des échanges. En fonction de chacun des événements évoqués, les énonciateurs ordonnent, composent, s'efforcent d'ajuster, dans l'interaction anticipée ou immédiate une mise en mots, chaque fois neuve (1995:5).

6 En nous interrogeant sur les pratiques d'écriture et ce qu'elles peuvent produire dans le cadre d'un audit organisationnel, nous souhaitons d'abord comprendre l'incidence du contexte, des conditions de productions sur la construction collective et négociée de sens. L'écriture est socialement située dans l'organisation investiguée mais également dans la relation entre les chercheurs, le commanditaire et les membres de l'organisation. En ce sens, le contexte contraint l'écriture et les acteurs dans leur négociation de l'écrit 
(Delcambre, 1990a ; Pène, 1995). Ensuite, nous nous interrogeons sur les stratégies de communication et d'expression des acteurs de l'écriture (Delcambre, 1990a) car « étudier les écrits, c'est prendre en compte la simultanéité des interactions humaines, c'est suivre la successivité du procès » (Pène, 1995 : 6). Cette analyse met en évidence la distribution du pouvoir/devoir d'écrire, inséparables de la forme des écrits. Plus particulièrement, nous nous interrogeons sur la place du chercheur-intervenant tenant « la plume » dans le dispositif d'écriture et dans les stratégies de communication des acteurs du système client.

7 Dans un second temps, cette théorie de la textualisation met en évidence l'action du texte (Brummans, 2007; Cooren, 2004). Une fois construit, le texte devient lui-même sujet à conversations mais, en tant que non-humain, il « participe également à l'émergence d'un ordre, non plus seulement social, mais socio-technique» (Cooren : 2010, 37). Dans une démarche d'intervention, cette "agentivité » est liée au dispositif de négociation. En effet, une fois écrit et remis au commanditaire, l'intervenant ne maitrise plus les effets du rapport. L'autonomie et la légitimité du rapport va donc, selon nous, dépendre du dispositif d'écriture mis en place et de la capacité des intervenants à tenir compte des stratégies d'acteurs, du contexte d'écriture, des destinataires et de sa finalité dans l'évolution de l'écrit. Il devra juger de ce qui peut ou non se retrouver dans le rapport tant sur le fond que sur la forme. C'est parce que le texte est négocié collectivement, légitimé et approprié par des acteurs de l'organisation qu'il peut potentiellement devenir un agent non humain agissant dans le processus d'amélioration de l'organisation.

\section{Contexte et matériaux analysés}

8 L'analyse proposée ici se fonde empiriquement sur de nombreuses expériences d'audit. Pour des raisons de place, nous nous focalisons sur la description d'un seul cas (récent) d'intervention en organisation particulièrement significatif des propositions analytiques que nous posons. L'intervention a concerné une institution du secteur associatif de la personne handicapée, composée de plus de 500 membres dans le personnel, qui depuis cinq ans, administre trois entités de taille très différente et anciennement autonomes pour des raisons historiques, fonctionnelles et juridiques, à savoir : un centre éducatif d'hébergement et de santé, un centre scolaire composé de trois structures distinctes, un centre de soins agréé. Chacune des entités dispose d'une structure hiérarchique propre, de règles administratives spécifiques et de modalités différentes (et indépendantes) de financement. Les activités principales de l'organisation se déroulent sur un site central. Cependant, hormis le plus petit centre, les autres entités développent aussi des actions à l'extérieur de ce périmètre, soit dans des lieux dédiés (une maison par exemple), soit en itinérance sur un territoire qui couvre deux Régions de la Belgique. Un conseil d'administration (CA) supervise ces trois entités avec un projet stratégique ambitieux qui met la personne handicapée au centre d'un travail professionnel collectif et multidisciplinaire et qui rend davantage poreuses les frontières multiples et rigides tant de la structure organisationnelle de chacune des entités que des logiques identitaires (de métier, de site...). Ainsi, un directeur général (qui est aussi le directeur d'une entité) assure la mission de gestion de l'ensemble de l'institution. Le projet a des difficultés à avancer, la structure complexe de l'institution et de ses entités ne facilite pas la tâche, ce qui justifie l'appel par le CA (le commanditaire) à une équipe de quatre intervenants universitaires spécialistes en organisation (dont les auteurs). Celle-ci a mis beaucoup de 
ressources humaines et d'énergie pour recueillir les données (plus de 150 interviews réalisées), les analyser et produire un rapport comportant des pistes concrètes d'actions, et qui s'est décliné en plusieurs versions.

Lors de cet audit, le processus d'écriture de ce rapport a joué un rôle clé. Le matériau s'est constitué par de fréquents retours vers la pertinence du terrain en faisant usage d'interviews - en cours de processus - orientées partiellement ou complètement « recherche " (vérification d'hypothèses empiriques) ${ }^{2}$. Cette construction s'est prolongée par une discussion du rapport de travail avec le CA suivie de réunions séquencées avec les directeurs des trois entités. Notons que le rapport final a été modifié entre chaque rencontre et une dernière fois avant la présentation finale au CA. La figure 1 ci-dessous retrace le cycle conversations-textes entre la première version du rapport et sa présentation finale au CA. On y retrouve dans le haut du schéma les conversations précisant les personnes avec qui les intervenants ont discuté ainsi que la focale de la discussion, et dans le bas, les quatre versions du rapport ainsi que l'évolution des genres d'écriture présents. Cette représentation cyclique met en évidence le caractère itératif du processus et son caractère collectif puisque les intervenants et les commanditaires coconstruisent le rapport. Il ne s'agit pas dans ce processus de trouver des solutions à tous les problèmes mais d'amener les commanditaires à prendre part à l'analyse.

Enfin, une autre forme de validation concerne l'équipe d'intervenants qui s'est organisée pour vérifier les connaissances produites et pour éviter les phénomènes de "sur » ou " sous » interprétation, d'instrumentation et d'influence par des témoins ou groupes de témoins, d'engagement $\mathrm{du}$ chercheur sans la nécessaire distanciation. Ainsi, trois réunions ont donné lieu à de l'herméneutique collective (Molitor, 1990) et le rapport final a fait l'objet de relecture par chacun et d'amendements négociés selon le principe de justification empirique ou théorique.

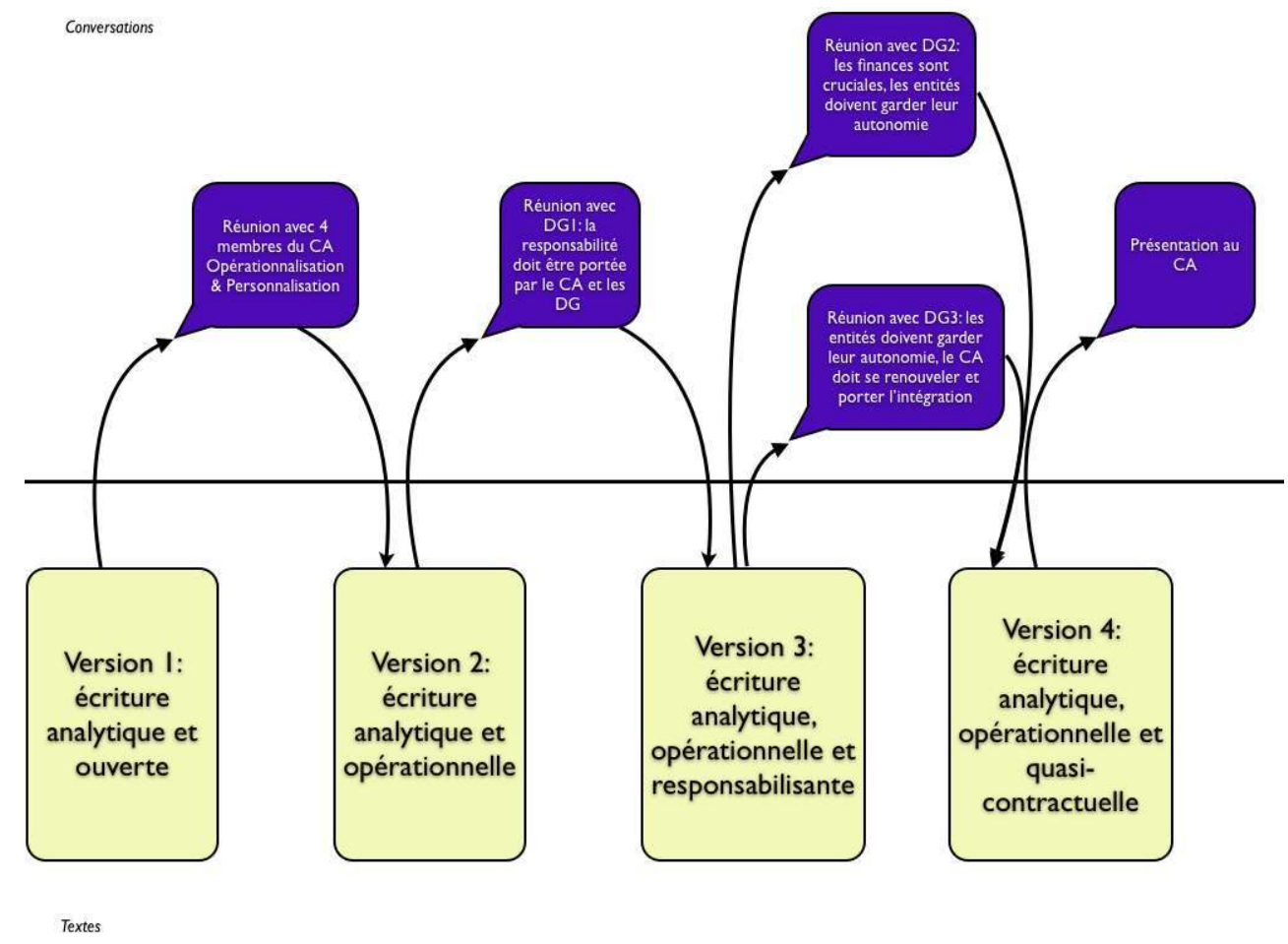

Figure 1 : Cycle texte-conversation reconstitué 
11 Dans un premier temps d'analyse, notre corpus de données se compose principalement des quatre versions du rapport à différent moments de l'audit. Nous disposons également de la convention de départ, des comptes-rendus de réunions, des présentations powerpoint faites auCA et d'un grand nombre de courriels échangés entre les intervenants d'une part et entre les intervenants et les commanditaires d'autre part. L'ensemble de ces documents permet de tracer longitudinalement l'évolution du rapport. La comparaison des quatre versions pointe les modifications qui y ont été réalisées. Celles-ci sont ensuite remises en contexte (Hansen, 2006 ; Pène, 1995) en revenant, par les comptes-rendus des réunions et les notes prises par les intervenants, sur les conversations au cours desquelles les intervenants ont discuté du rapport et de son contenu avec les commanditaires et les trois directeurs. Le temps écoulé a favorisé l'analyse à froid des traces écrites et une distanciation critique aux données.

Dans un second temps, nous analysons les échanges de courriel et les documents émis par le président du CA qui ont suivi la fin de l'intervention et qui témoignent d'une certaine forme d'agentivité du rapport. C'est à travers cette analyse que nous questionnons d'une part le sens pris par notre intervention à travers le rapport dans la relation entre le CA et les trois directeurs et que nous montrons d'autre part l'importance de la textualisation dans la légitimation du rapport. Précisons que nous parlons de légitimité auprès des membres du CA et de la direction et non auprès des employés. Le rapport se base principalement sur le recueil des témoignages des employés et tente d'identifier les problèmes tels qu'ils sont envisagés par ceux-ci et pas nécessairement par la direction. Qui plus est, le diagnostic posé met en avant un problème de gouvernance au sommet de la hiérarchie. Il était donc avant tout nécessaire de convaincre le CA et les directeurs qu'ils faisaient partie des problèmes qu'ils devaient résoudre par eux-mêmes. Nous vérifions aussi l'hypothèse, dans une perspective constructiviste interactive, de l'informalité inhérente au rapport d'audit, soumis à interprétations, réinterprétations et appropriations sociocognitives, parce que sa nature même est compréhensive et pas seulement instrumentale.

\section{Résultats et interprétations}

\section{Analyse du dispositif d'écriture : de l'écriture analytique à l'écriture quasi-contractuelle}

Les résultats du premier temps d'analyse montrent une transformation du contenu à la suite d'une addition séquentielle de fonctions au document. Ainsi, la version $1 \mathrm{du}$ rapport a comme objectifs d'une part la présentation de l'analyse de l'organisation et des modèles qui servent de cadre théorique et d'autre part une première validation de l'étude par les commanditaires. L'analyse, d'ordre systémique, repose sur un principe éthique (négocié avec les commanditaires et les témoins interviewés) (Scieur et al., 2008) qui n'engage ni ne mentionne les acteurs. Relevant ainsi d'une perspective strictement fonctionnelle (une réponse formalisée à une demande ou à un besoin), l'étude menée peut satisfaire intellectuellement les quatre membres du CA rencontrés lors de la première réunion (qui dure quatre heures), ce qui en constitue une première validation de leur part. Cependant, ils sont très conscients de leur rôle au sein de l'organisation ${ }^{3}$ et se positionnent sur un plan stratégique (au sens managérial). Cela conduit à faire émerger deux exigences communicationnelles complémentaires : la synthèse et l'opératoire. Une autre exigence 
émerge du dialogue avec le commanditaire, la personnalisation, qui met à la fois en cause les engagements éthiques de l'intervention (cf. supra) et en tension conflictuelle les acteurs de la textualisation.

Dans la version 2 du rapport, à l'analyse, s'ajoute une dimension opérationnelle qui se marque par trois types de modifications textuelles :

(1) l'usage de concepts interactionnistes comme par exemple la traduction : « Les commissions d'admission relèvent de cette difficulté de traduction, entre les différents professionnels d'une part, et entre les objectifs définis par le CA au regard du profil des résidents et la manière dont ils sont perçus par les responsables de terrain d'autre part »; (2) l'élaboration d'une synthèse de cinq points forts de l'institution et de cinq axes de progrès, parfois maillés, qui témoigne d'un souhait par le commanditaire, en déterminant les principaux axes clés organisationnels positifs et négatifs, de réduire la complexité analytique et d'intégrer dans le document une dimension normative (évaluative) : « le plan d'actions mis en œuvre pour rassembler les équipes autour du projet institutionnel a permis de réels changements ";

(3) la constitution de deux scénarios de gouvernance basés sur des principes très structurants, susceptibles de controverses, qui conduisent à des idéaux-types configurationnels d'intégration des entités et des services. Le premier, qualifié de réaliste en fonction de la situation analysée, propose un niveau d'intégration des fonctions de service (en staff) en laissant le leadership aux départements opérationnels ; le second, qualifié d'idéaliste, renverse la matrice fonctionnelle et met en avant la structure des services et leur responsable, avec une intégration maximale des entités alors complètement transversalisées. Ces deux modèles, politiques, sont l'objet principal des discussions avec les trois directeurs généraux et entrainent la construction des versions suivantes du texte comme cela est illustré dans les figures deux et trois ci-après.

La version $3 \mathrm{du}$ rapport repose sur la relecture par le directeur général de l'institution $(D G 1)^{4}$. Cette version met en évidence une nouvelle préoccupation, celle de la responsabilité dans le modèle de gouvernance envisagé et de sa traduction communicationnelle, dans les schémas et dans le texte. La compréhension de la lecture du DG1 s'inscrit dans le contexte organisationnel diagnostiqué mais aussi dans celui du processus d'intervention, c'est-à-dire de la commande et ses motivations. En effet, le "premier » responsable opérationnel de l'institution considère sa légitimité atteinte par l'appel à des auditeurs externes même si intellectuellement, il considère que cette opération peut apporter une plus-value à la gestion de son institution. Pour lui, le CA et les autres DG le considèrent comme responsable des difficultés organisationnelles. Il propose donc, sans remettre en cause l'analyse et le texte de la version 2, de revoir le schéma de gouvernance envisagé, en intégrant au niveau opérationnel de la direction générale (le comité de direction), en plus de ses collègues, trois administrateurs désignés par le CA. Cette proposition vise à faire porter la mise en œuvre opérationnelle des choix stratégiques aussi par le $\mathrm{CA}$ et à collégialiser les décisions. Une nouvelle tension se révèle, entre les membres du CA et le DG1, autour de trois considérations managériales de la responsabilité: la personnalisation (l'attente duCA), la collectivisation (le souhait duDG1), ou la fonctionnalisation systémique (la modalité conventionnelle de l'intervenant). Cette tension se traduit par des visualisations graphiques différentes et des commentaires discordants entre cette version et la suivante comme les traces de modifications du rapport le montrent dans les figures deux et trois repris ci-dessous. 
Version 2

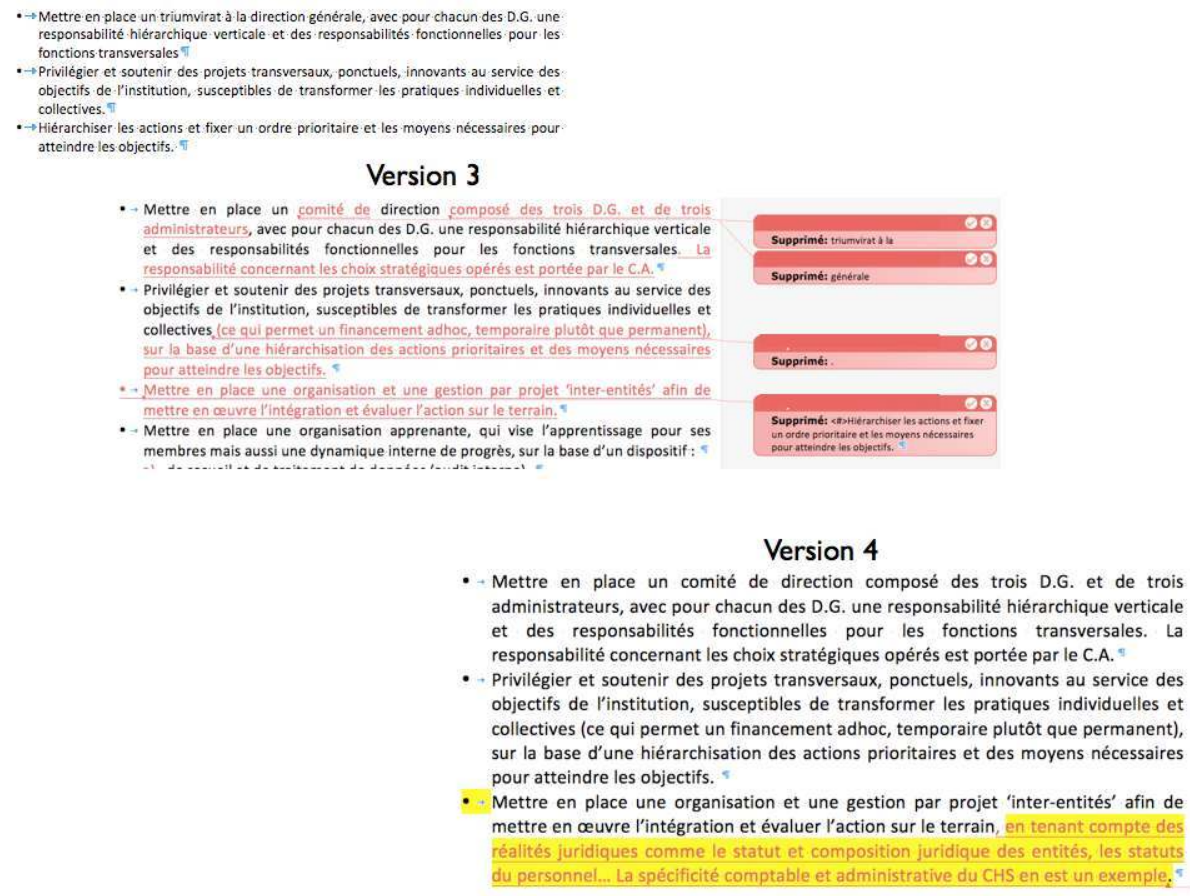

Figure 2 : tracé des modifications des "principes de bases »

\section{Version 2}

- Un triumvirat à la barre avec des missions ou des projets transversaux et circonscrits.

- CDI doit servir à établir les politiques et les directions générales vers la hiérarchie

intermédiaire.

- BE-CDI est en phase test et devra être plus opérationnel (problème d'absentéisme de

certains) si l'on veut qu'il gagne en efficacité."

\section{Version 3}

- Un comité de direction à la barre avec des missions ou des projets transversaux et circonscrits.

$\cdots$ Ce comité de direction aurait à sa charge le pilotage de l'organisation, la mise en ceuvre des objectifs à savoir l'élaboration de choix stratégiques et la validation des plans d'actions y afférant. "

$\rightarrow-$ Pour chaque grand projet transversal, un DG serait le référent. II serait entouré d'un comité de pilotage composé de membres du CA mais aussi le cas échéant d'experts externes (en fonction du projet). 5

- - De la dénomination du DG référent: il revient au CA de nommer le DG responsable du projet. '

$\rightarrow$ La responsabilité d'un projet n'implique pas nécessairement que le DG soit à la manoeuvre pratiquement. II peut, si nécessaire (et c'est recommandé), désigner un chef de projet (dont c'est le domaine d'expertise et pas nécessairement dans son entité) en charge de sa mise en œuvre sur le terrain. "

- - Le CDI disparaít. Les D.G. relaient les politiques et les directions générales vers feurhiérarchie intermédiaire. 5

- Le BE-CDI reste en place avec des objectifs purement opérationnels de gestion du quotitien inter-entités. Mais, le BE-CDI est en «phase test » et devra être plus opérationnel (problème d'absentéisme de certains) si l'on veut qu'il gagne en efficacité. "

\section{Version 4}

* Veiller à maintenir une dynamique au sein du C.A. à moyen et long termes, notamment par le recrutement à venir:

Un comité de direction à la barre avec des missions ou des projets transversaux et circonscrits."

- Ce comité de direction aurait à sa charge le pilotage de l'organisation, la mise en œuvre des objectifs à savoir l'élaboration de choix stratégiques et la validation des plans d'actions y afférant. "

Figure 3 : tracé des modifications des "propositions d'actions en matière de gouvernance »

La dernière version du document repose sur le feedback rendu par deux autres directeurs généraux de l'institution (DG2 et DG3). Deux changements ont été effectués. L'un concerne la mise en place d'une organisation par projet inter-entité, qui doit se faire en respect strict du statut juridique de chacune des entités. L'autre insiste sur la nécessité de maintenir une dynamique au sein du CA sur les moyen et long termes. Ces modifications 
traduisent les préoccupations des DG mais aussi le niveau organisationnel qu'ils considèrent comme prioritaire pour mettre en œuvre une nouvelle politique institutionnelle. La version écrite finale acquiert une nouvelle dimension, celle de la quasi-contractualisation dont le texte constitue le garant formel. Sur le plan théorique conventionnel (Boltanski et Thévenot, 1991), l'aboutissement de la co-construction du rapport d'audit, à la suite de négociations et de controverses tranchées, conduit à poser un "monde" (une cité) hybride dont le principe supérieur commun repose sur un maillage du civisme, de la domesticité et de la productivité. La textualisation opérée constitue alors une mise à l'explicite des tensions qui traversent l'organisation et des choix pour les gérer.

17 En résumé, là où les premiers changements visent l'opérationnalisation et la gouvernance, les changements opérés en versions 3 et 4 avaient plus comme vocation d'intégrer au rapport les conditions d'acceptation des priorités et les conditions d'engagement des directeurs généraux dans la nouvelle gouvernance. C'est ainsi que le travail d'audit s'est transformé petit à petit en une forme de contrat formel dont la première partie a posé le contexte et les problèmes et la deuxième sa mise en œuvre sous conditions. Les intervenants extérieurs sont en quelque sorte devenus des médiateurs entre les membres du conseil d'administration et les directeurs généraux.

\section{De l'agentivité du rapport}

18 Si notre mission s'est clôturée lors de la présentation du rapport au conseil d'administration, plusieurs documents envoyés par courriel et les courriels eux-mêmes montrent la volonté du président du CA de légitimer son action en s'appuyant sur le rapport. Dans un premier document, le président reprend mot à mot des éléments du rapport dans sa communication vers les employés. Les parties reprises sont celles dites opérationnelles du rapport. Elles sont suivies d'une série de décisions du CA lors d'une réunion ayant eu lieu (un mois et demi) après la présentation du rapport au $\mathrm{CA}$. Ces décisions tiennent en cinq points: le premier est une confirmation de la volonté d'intégration des entités de l'organisation, le deuxième est la mise en œuvre (partielle) du principe de gouvernance préconisé dans le rapport, les trois derniers points sont des intentions (nous examinerons ou avons examinés) mais qui ne débouchent sur aucune mise en œuvre.

Dans un second courriel, le président du CA se réfère à deux reprises au rapport de façon explicite. Il y est fait référence une première fois pour donner sens au départ précipité de DG1 de l'institution dans les mois qui ont suivi l'audit: « DG1 pensait que le 'rapport' lui dirait simplement comment diriger son entité. Il n'était pas préparé du tout au diagnostic fondé sur les réactions de personnels et ne s'attendait pas à ne pas être renommé directeur général ». Puis une seconde fois, pour mentionner DG2 qui a repris la direction générale et qui était assez sceptique concernant certains de nos diagnostics mais que sa propre analyse du terrain vient renforcer : «DG2 passe un temps considérable à rencontrer le personnel de l'entité d'éducation et se rend compte de nombreux problèmes qui confirment ce que vous aviez décrit dans votre rapport ».

Il est intéressant de voir qu'il est fait, dans les deux cas, référence au rapport concernant l'acceptation des diagnostics posés par l'intervenant. Rappelons que cette partie analytique est celle qui n'a pas fait l'objet de modifications majeures mais qu'elle est le socle de l'opérationnalisation et de la responsabilisation ayant fait l'objet de toutes les 
réunions. En ce sens, on peut dire que le rapport agit en imposant les analyses aux directeurs généraux qui ne peuvent les ignorer puisqu'elles forment la base des actions négociées avec eux. Deux stratégies s'offrent alors aux acteurs ne souhaitant pas mettre en œuvre les propositions négociées : l'une consistant à refuser le diagnostic et à partir (ce que fera finalement le DG1) et l'autre consistant à le valider ou l'invalider soi-même. Le rapport prolonge l'intervention, presque physiquement (Pène, 1995) et agit sur le travail des membres de la direction et du CA qui s'y réfèrent pour appuyer certaines décisions comme pour expliquer les réactions du DG1 ou celles de DG2. Il est devenu le garant du contrat passé entre le CA et la direction. Nous pensons que la co-construction par l'écriture négociée mais également l'évolution du style de l'écrit traduisant le contexte organisationnel et intégrant les stratégies d'expression des acteurs ont permis de légitimer son contenu et d'en faire un actant de l'organisation et de son fonctionnement.

\section{L'approche constructiviste, plus qu'une idéologie d'intervention}

Notre propos s'inscrit pleinement dans une approche épistémologique constructiviste en mobilisant mais aussi en interrogeant ses principes fondateurs ${ }^{5}$. Jean-Louis Le Moigne (1995) propose quatre principes que nous présentons comme les fondements d'une intervention socio-constructiviste. Le premier évoque la connaissance qui ne peut avoir de sens ou de valeur en dehors du sujet connaissant. Le travail de textualisation révèle ce lien communicationnel et cognitif interactif et permanent entre les acteurs concernés par la rédaction et la compréhension du rapport d'audit, objet finalement des transactions cognitives. Le cas présenté ici est particulièrement significatif de cette co-construction par les différents acteurs concernés d'un rapport final qui est la quatrième version d'un texte dont le fondement analytique n'est jamais remis en cause au cours du processus d'intervention, mais qui évolue par ce que l'on peut appeler des négociations de sens. Audelà de son agentivité processuelle (cf. supra), le document acquiert ainsi in fine un statut légitime de cadre conventionnel d'action auprès des commanditaires de l'étude.

Le réel n'est donc connaissable que par l'expérimentation des sujets (deuxième principe) qui autour de cet objet de transaction mobilisent leurs schèmes mentaux, sociaux et culturels et les mettent collectivement en confrontation. La lecture argumentée de l'organisation que constitue l'analyse effectuée opère, nous l'avons montré, chez les acteurs concernés de l'institution comme un incitant à la mise en distanciation de leur expérience organisationnelle. Les moments de confrontation des points de vue des acteurs concernés constituent des opportunités de faire évoluer le texte et de clarifier (et complexifier) pour le commanditaire sa demande initiale comme l'illustre le schéma 1.

La méthodologie pour saisir la complexité du système interactionnel qui se construit est systémique (troisième principe). Le processus de textualisation agit par système de bouclage et de rétroaction, ce qui lui assure un caractère évolutif. Sur le plan épistémologique, l'analyse se situe au niveau du système (fonctionnalisme), sans nier qu'il est constitué par des acteurs en interaction (analyse stratégique) situés dans un contexte particulier (analyse culturaliste). Cependant, la démarche est inductive car les modèles théoriques mobilisés permettent l'élaboration d'un diagnostic organisationnel pluriel qui s'affine et gagne en robustesse à chaque étape de validation, à chaque période de 
confrontation et de mise à l'épreuve par les acteurs concernés de l'institution étudiée (Scieur et al., 2008).

La méthodologie s'accompagne épistémologiquement d'une dimension éthique (quatrième principe) dans l'inscription de la démarche d'intervention dans un cadre socioculturel de valeurs. Par exemple, sur le plan du processus, la remise en question par le commanditaire, entre la phase 1 et la phase 2 surtout, de la non-personnalisation du rapport devient un objet de tension éthique, dans la mesure où la convention d'intervention acceptée par les parties l'exclut d'une part, et que cet accord est explicitement mentionné au personnel lors des rencontres préparatoires de présentation du dispositif d'autre part. A titre illustratif, sur le fond, la finalité politique de l'institution, orientée vers le bien-être du résident, selon une approche globale et pluridisciplinaire, a constitué très clairement un cadre valoriel qui a influencé les propositions opérationnelles des versions 2, 3 et 4 du rapport.

L'intervenant en organisation assure donc une mission originale: il est médiateur, épistémologue, en charge de discuter à la fois les hypothèses qui génèrent les connaissances mais aussi de délibérer sur leurs fondements éthiques ${ }^{6}$. Ainsi, il met à l'épreuve l'ensemble de sa démarche en définissant une perspective qui, selon Ricoeur (1990), peut se définir par la visée de la vie bonne, avec et pour les autres, dans des institutions justes. Mais cette visée n'est pas donnée a priori, elle s'élabore partiellement dans l'interaction avec le commanditaire (notamment dans la co-écriture du rapport) et surtout dans l'opérationnalisation des conclusions de l'audit qui révèlent concrètement les valeurs des acteurs concernés. Cette dernière remarque souligne l'opportunité de prendre en considération une épistémologie constructiviste qui dépasse (tout en l'intégrant) une acception individualiste, que l'on retrouve chez Mucchielli (2004), et valorise une perspective interactionniste, autour du processus de co-construction d'un sens commun.

\section{Pour conclure}

De façon réflexive, nous concluons par la genèse de l'article et ses apports. D'abord, l'article lui-même est le fruit d'une fertilisation croisée entre pratiques et théories. Philippe Scieur pratique l'audit organisationnel depuis plusieurs années et a fortement insisté sur le dispositif de négociation à mettre en place pour finaliser le rapport. François Lambotte, qui réfléchit sur les modèles d'audit de communication interne, était peu convaincu par les explications existantes concernant « l'après » audit. Cette rencontre de terrain a permis a posteriori aux deux chercheurs une mise en commun de leurs réflexions tant théoriques que pratiques éclairant la dimension critique de la négociation et de la transmission du rapport d'audit des mains de l'auditeur à celles du commenditaire.

Cette recherche réfléchit ce passage comme un processus de co-construction, à travers le cycle texte-conversation et montre que le rapport et ses recommandations ne sont pas désincarnés. Ainsi, il est possible de tenir compte du caractère social et négocié de la signification dans la pratique même de l'audit faisant du socio-constructivisme plus qu'une simple idéologie. 


\section{BIBLIOGRAPHIE}

Anderson D. L., (2004), The Textualizing Functions of Writing for Organizational Change, in Journal of Business and Technical Communication, vol. 18, $\mathrm{n}^{\circ}$ 2, pp. 141-164.

Boltanski L. et Thévenot L., (1991), De la justification. Les économies de la grandeur, Paris, Gallimard, 483 p.

Brummans B. H. J. M., (2007), Death by Document : Tracing the Agency of a Text, in Qualitative Inquiry, vol. 13, $\mathrm{n}^{\circ}$ 5, pp. 711-727.

Cooren F., (2004), Textual Agency: How Texts Do Things in Organizational Settings, in Organization, vol. $11, \mathrm{n}^{\circ} 3$, pp. 373-393.

Cooren F., (2010), Ventriloquie, performativité et communication, in Réseaux, vol. 163, n 5, pp. 33-54.

Delcambre P., (1990a), Une analyse d'écriture revisitée : ou, quand l'analyse d'écriture fait intervention, que dire de ses effets?, in Études de communication, $\mathrm{n}^{\circ} 11$, pp. 131-150.

Delcambre P., (1990b), Écritures professionnelles et pouvoirs : Analyse d'un dispositif d'écriture, in Études de communication, $\mathrm{n}^{\circ} 11$, pp. 13-53.

De Saint-Georges P., (2000), La « formation-symptôme » intervention et changement dans les organisations, in Communication et Organisation, $\mathrm{n}^{\circ} 17$, pp. 194-216.

Fayad F. et Lambotte F., (2011), Le sensemaking collectif dans une équipe virtuelle, in Recherches en Communication, $\mathrm{n}^{\circ} 33$, pp. 131-146.

Friedberg E., (1993), Le Pouvoir et la règle. Dynamiques de l'action organisée, Paris, Seuil.

Hansen H., (2006), The ethnonarrative approach, in Human Relations, vol. 59, n 8, pp. 1049-1075.

Kuty O. et Vrancken D. (dir.), (2001), La Sociologie et l'Intervention : Enjeux et Perspectives, Bruxelles, De Boeck, 357 p.

Lambotte F. et Lafrance A. A., (2011), Tenir le C.A.P. de vos communications en entreprise : pour une performance optimale de vos réseaux humains et techniques, Liège, Edipro, 175 p.

Le Moigne J.-L., (1995), Les épistémologies constructivistes, Paris, PUF, 128 p.

Molitor M., (1990), L’herméneutique collective, in Remy J. et Ruquoy D. (dirs.), Bruxelles, éditions des Facultés Universitaires Saint-Louis, pp. 19-29.

Mucchielli A., (2004), Approche par la modélisation des relations : Études des communications, Paris, Armand Colin, $192 \mathrm{p}$.

Mucchielli A., (2005), Information et communication interne : Étude des communications. Pour de nouveaux audits ( $2^{\mathrm{e}}$ éd.), Paris, Armand Colin, $205 \mathrm{p}$.

Pène S., (1995), Les écrits et les acteurs. Circulation des discours et empreinte des objets, in Études de communication, $\mathrm{n}^{\circ} 16$, pp. 57-75.

Ricoeur P., (1990), Soi-même comme un autre, Paris, Seuil, 424 p.

Robichaud D. et Benoit-Barné C., (2010), L'épreuve de la conversation : comment se négocie la mise en œuvre des normes dans l'écriture d'un texte organisationnel, in Études de communication, $\mathrm{n}^{\circ} 34$, pp. 41-60. 
Robichaud D., Giroux H. et Taylor J. R., (2004), The metaconversation : the recursive property of language as a key to organizing, in Academy of Management Review, vol. 29, n 4, pp. 617-634.

Scieur P., Mahieu C. et Vanneste D., (2008), La responsabilité sociale au regard des exigences épistémiques et éthiques de la recherche et de l'intervention sociologique en organisation, in Pichault F. et Lisein et al. (dirs.), La recherche-intervention peut-être être socialement responsable ?, Paris, Vuibert, collection AGRH, pp. 201-217.

Scieur P., (2011a), Sociologie des organisations. Introduction à l'analyse de l'action collective organisée, 3 e éd., Paris, Armand Colin, 188 p.

Scieur P., (2011b), Quelles exigences épistémologiques pour l'analyse organisationnelle? Vers la production scientifique de connaissances au cœur de la complexité, in de Nanteuil M. et Taskin L. (dirs.), Perspectives critiques en management. Pour une gestion citoyenne, Bruxelles, De Boeck, pp.

121-134.

Taylor J. R., Cooren F., Giroux N. et Robichaud D., (1996), The Communicational Basis of Organization: Between the Conversation and the Text, in Communication Theory, vol. 6, $\mathrm{n}^{\circ}$ 1, pp. 1-39.

\section{NOTES}

1. Notons cependant en sociologie quelques références comme Friedberg (1993), Kuty et Vrancken (2001), Scieur (2011a).

2. L'hypothèse se conçoit ici comme une proposition analytique évolutive qui, produite au début $\mathrm{du}$ processus de recherche par le rapport empirique du chercheur à son objet, prend au fur et mesure de l'avancement du dispositif et des étapes méthodologiques, de la robustesse sur le plan de l'intelligibilité scientifique.

3. Notre expérience du monde associatif montre que le rôle du CA est, au-delà des prescrits légaux en la matière (relativement élémentaires), une construction sociale souvent endogène, fruit d'interactions et de facteurs historocistes.

4. Rappelons-le, le DG de l'institution est aussi le DG de l'entité la plus importante (en termes de nombre de membres du personnel). Le DG2 et le DG3 sont responsables des deux autres entités.

5. Pour un développement de cette approche au regard des théories organisationnelle, cf. Scieur 2011b.

6. Voir notamment Scieur, Mahieu et Vanneste, 2008.

\section{RÉSUMÉS}

Cet article porte sur le statut du rapport dans l'audit organisationnel. A travers l'analyse du dispositif d'écriture mis en œuvre lors d'une intervention récente, il met l'accent sur (1) la construction de la légitimation de l'audit à travers son écriture, fruit d'une construction interactive entre les acteurs concernés par la convention, et sur (2) son rôle d'interactant non humain, au sens de la sociologie de la traduction, qui prolonge l'interaction entre les acteurs temporellement au-delà de la remise finale du rapport au commanditaire. Il questionne la place 
du chercheur dans l'intervention et montre comment l'approche socio-constructiviste s'inscrit pleinement dans l'intervention.

This paper addresses the status of the report in organizational auditing. Through the analysis of the textualization taking place in a recent intervention, it first emphasizes the construction of legitimacy in an audit's results as the product of the interaction among actors concerned by the conventions involved, and then its role as a nonhuman interactant as defined in the sociology of translation, which extends the interaction among actors temporally beyond the submission of the final report to the client. The paper questions the place of the scientist in the intervention and shows how a socio-constructivist approach is fully part of the process.

\section{INDEX}

Mots-clés : écriture, textualisation, audit, rapport, légitimité, socio-constructivisme

Keywords : writing, textualization, audit, report, legitimacy, socio-constructivism

\section{AUTEURS}

\section{FRANÇOIS LAMBOTTE}

LASCO - site de Mons, Université Catholique de Louvain

François Lambotte est docteur en sciences de gestion, professeur à l'école de communication de l'université catholique de Louvain à Mons (Belgique). Il est vice-président du laboratoire d'analyse des systèmes de communication des organisations (LASCO). Adresse électronique : francois.lambotte@uclouvain-mons.be.

\section{PHILIPPE SCIEUR}

CRIDIS - site de Mons, Université Catholique de Louvain

Philippe Scieur est sociologue, professeur à l'université catholique de Louvain à Mons (Belgique). Il est chercheur au centre de recherche interdisciplinaire Démocratie, Interventions, Subjectivité (UCL/CriDIS) et directeur du Centre de recherche et d'intervention en organisation (UCL Mons/CeRIO). Adresse électronique : philippe.scieur@uclouvain-mons.be. 